\title{
COMPOSICIÓN FÍSICO QUÍMICA Y ANÁLISIS PROXIMAL DEL FRUTO DE SOFAIQUE Geoffroea decorticans (Hook. et Arn.) PROCEDENTE DE LA REGIÓN ICA-PERÚ
}

\author{
Carlos J. Pillco Cochan*, Deysi Guzmán Loayza ${ }^{a}$, José E. Cuéllar Bautista ${ }^{a}$
}

\begin{abstract}
RESUMEN
La presente investigación evaluó el fruto de Geoffroea decorticans (Hook. et Arn.) procedente del desierto costero en el departamento de Ica (Perú), conocido con el nombre de "Sofaique" y en otras regiones limítrofes del país con el nombre de "Chañar". Se tuvo como objetivo principal dar conocimiento sobre su composición fisicoquímica y nutricional; la muestra fue extraída del fruto de forma manual donde se separó en epicarpio, mesocarpio y carozo; de las tres fracciones solo se analizó el mesocarpio utilizando muestras de $3 \mathrm{~g}$ para los ensayos químicos (acidez titulable, $\mathrm{pH}$, sólidos solubles) y nutricionales (humedad, proteínas, lípidos, minerales y carbohidratos) en base a los métodos descritos por la AOAC (Association of Offical Analytical Chemistry), para la caracterización física se realizó la medición de las dimensiones del fruto entero en sus 3 ejes (polar, ecuatorial y calibre) utilizando un vernier digital y el cálculo del peso mediante una balanza analítica. El resultado promedio de las dimensiones del fruto entero fueron: eje ecuatorial $22,32 \mathrm{~mm}$, eje polar $21,69 \mathrm{~mm}$ y calibre $19,60 \mathrm{~mm}$; el promedio de las proporciones del fruto, fueron: pulpa $38,51 \%$, carozo 32,91 $\%$ y cascarilla 28,58\%; y el peso promedio del fruto entero fue de $3,88 \mathrm{~g}$; los principales resultados del análisis proximal y composición química del mesocarpio (pulpa) fueron 9,17 $\%$ de proteína; 2,59 \% de cenizas; 20,20\% de contenido de humedad; 1,38 \% de lípidos; $66,66 \%$ de carbohidratos; 5,2 de $\mathrm{pH} ; 0,05 \%$ de acidez titulable y 4,0 $0^{\circ}$ Brix en sólidos solubles. Con los resultados expuestos se concluye que el fruto de sofaique resulta ser un fruto nutritivo y agradable al paladar, teniendo en cuenta que es una especie que se desarrolla en zonas semiáridas y áridas, donde los nutrientes disponibles en el suelo son muy limitados.
\end{abstract}

Palabras clave: Geoffroea decorticans, análisis proximal, nutricional, biometría, sofaique.

\footnotetext{
${ }^{a}$ Facultad de Ciencias Forestales, Universidad Nacional Agraria La Molina, Av. La Molina s/n, Lima, Perú, cpillco123@gmail.com.
} 


\title{
PHYSICAL CHEMICAL COMPOSITION AND PROXIMAL ANALYSIS OF THE FRUIT OF SOFAIQUE Geoffroea decorticans (Hook. et Arn.) FROM THE ICA-PERU REGION
}

\begin{abstract}
The current study evaluated Geoffroea decorticans (Hook. et Arn.) fruit, from the coastal desert in the department of Ica (Perú), well known by the name of "Sofaique" and in other regions "Chañar". The main objective of this research was to obtain data about its physicochemical and nutritional composition; the sample was extracted of fruit and was separated into three parts: epicarp, mesocarp and pit. The AOAC methodology was applied in order to determine its chemical and nutritional properties using only the mesocarp with samples of 3 grams each. For the physical characterization, the fruit dimensions were measured in its three axes (polar, equatorial and caliber) with a digital caliper and the weight by an analytical weighting scale. The average results of the whole dimensions of fruit were: equatorial axis $22,32 \mathrm{~mm}$, polar axis 21,69 $\mathrm{mm}$ and caliber 19,60 $\mathrm{mm}$; the average of the fruit proportions were: pulp $38,51 \%$, stone $32,91 \%$ and peel $28,58 \%$, and the average weight of the whole fruit was $3,88 \mathrm{~g}$; the main results of the proximal analysis and chemical composition for mesocarp (pulp) were 9,17 \% protein; 2,59\% ashes; 20,20\% moisture content; 1,38 \% lipids; $66,66 \%$ carbohydrates; $\mathrm{PH} 5,2 ; 0,05 \%$ titratable acidity and $4,0^{\circ}$ Brix in soluble solids. The results indicate that "sofaique" turns out to be a nutritious and palatable fruit, considering that it is a species that grows in semi-arid and arid areas with limited nutrients available in soil.
\end{abstract}

Key words: Geoffroea decorticans, proximal analysis, nutritional, biometrics, sofaique.

\section{INTRODUCCIÓN}

Geoffroea decorticans conocida popularmente como "Sofaique", es una especie arbórea arbustiva perteneciente a la familia de las Fabaceaes, situada en el departamento de Ica (Perú), en otras regiones limítrofes del país se conoce con el nombre de "Chañar"; crece habitualmente en los grandes desiertos costeros pudiendo extenderse hasta los $2500 \mathrm{msnm}$, además suele agruparse como bosquecillos o rodales puros, y también en asociación con algunas palmeras xerófilas. En el desierto costero del Perú específicamente en la región de Ica, la fructificación empieza a finales del mes de diciembre hasta inicios del mes de marzo, donde se puede apreciar frutos de color pardo-rojizo en la copa del árbol y sobre el suelo con tonalidades más oscuras.

El árbol de sofaique es un recurso natural poco conocido y sin algún tipo de uso por la población en las diferentes regiones, esto se debería a la escasa investigación del aprovechamiento sustentable de los recursos que puede brindar la especie, en otros países como Chile, Argentina o Paraguay, los estudios con respecto a esta especie han ido en 
incremento tanto para el fruto como para otras partes vegetativas, debido a que es considerada una especie multipropósito con buena adaptabilidad a condiciones limitadas de sitio; como se mencionó antes, el sofaique habita en los grandes desiertos costeros áridos y per-áridos como bosquecillos, esta asociación se debe al nulo o escaso consumo de las semillas, las cuales logran germinar en el mismo lugar, además de ello presentan raíces gemíferas, esta última característica ha ocasionado que algunos pobladores consideren a la especie como una plaga a causa de su rápido desarrollo y comportamiento agresivo.

Reflexionando sobre la importancia e insuficiente información respecto a la especie $G$. decorticans, se realizaron investigaciones en materia de composición química y nutricional en diferentes países, según McCance y Widdowson ${ }^{1}$ mencionan que el conocimiento de la composición química de los alimentos es el primer elemento esencial en el tratamiento alimentario de las enfermedades o en cualquier estudio cuantitativo de la nutrición humana.

La presente investigación tiene como objetivo evaluar el fruto del sofaique, G. decorticans, en su composición fisicoquímica y nutricional, determinando así su importancia y valor potencial como alimento nutritivo, tanto para personas en el consumo directo o procesado y para animales como producto forrajero, asimismo, ser considerada una especie promisoria para ser usada dentro de dietas para los ganados vacuno y porcino, especialmente.

\section{PARTE EXPERIMENTAL}

\section{Descripción de la zona de estudio}

El bosquecillo de sofaique se encuentra en medio del desierto de Paracas, junto a otras especies en menor proporción como son: palmeras datileras (Phoenix dactylifera), árboles de charán (Caesalpinia paipai Ruiz \& Pavón) y algunos pastos eriazos; donde se destaca un paisaje desolado sin ningún otro tipo de vegetación natural circundante, pero sí apreciando cultivos agrícolas como: uva, espárragos, olivo, algodón, entre otros, instalados aproximadamente a 3 kilómetros del bosque relicto; asimismo, este se encuentra a 16 kilómetros del río Pisco el cual es el principal regulador de la napa freática. El INRENA ${ }^{2}$ clasifica a esta zona como un desierto desecado-Subtropical (dd-S), donde el escenario muestra suelos de textura variable, entre ligeros a finos, con cementaciones salinas, cálcicas o gipsicas (yeso) y con incipiente horizonte A superficial con menos de $1 \%$ de materia orgánica.

\section{Descripción de la muestra}

Se seleccionaron cinco árboles al azar, de los cuales se colecto un total de $3 \mathrm{~kg}$ de muestra (frutos), la colecta fue únicamente de frutos maduros en buen estado (figura 1), tanto de la copa como del suelo, estos individuos se encuentran localizados en el asentamiento humano Las Antillas, distrito Paracas, departamento Ica. 


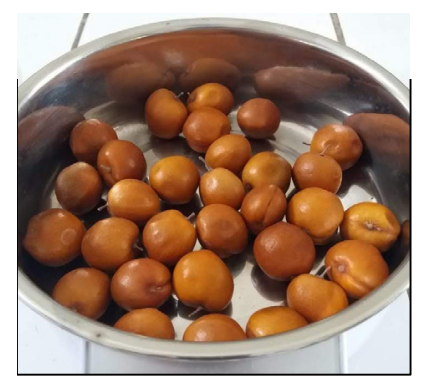

Figura 1. Frutos maduros en buen estado.

Fuente propia

\section{Preparación de la muestra}

Los frutos después de haber sido almacenados a una temperatura bajo cero, fueron retirados y mezclados en un solo envase logrando homogeneizar la muestra. Se seleccionó una muestra de 30 frutos al azar para realizar las medidas biométricas, que constó de la medición de sus tres ejes dimensionales para posteriormente separar el epicarpio (cascarilla) del mesocarpio (pulpa), y los mismos del carozo de forma manual haciendo uso de una pequeña cuchilla y espátula. El siguiente procedimiento fue separar cada uno de los componentes (epicarpio, mesocarpio y carozo) en distintos envases para proseguir con el análisis de la composición química y proximal.

\section{Métodos}

Para las pruebas físicas, como peso de fruto, se utilizó una balanza analítica OHAUS de $0,1 \mathrm{mg}$ de precisión, para la medición del eje polar, ecuatorial y calibre se utilizó un pie de rey digital Vogel, en los análisis químico y proximal se utilizaron las metodologías de la Association of Offical Analytical Chemistry ${ }^{3}$; para todas las variables estudiadas se realizaron tres repeticiones:

Acidez titulable (Método AOAC 942.15): Luego de homogenizar la muestra, se coloca 2 $\mathrm{g}$ de pulpa en un matraz agregando $100 \mathrm{~mL}$ de agua destilada, se deja hervir por una hora, reponiendo el agua perdida por evaporación, se enfría y trasvasa a una fiola de $500 \mathrm{~mL}$ se agita y se deja en reposo, de la filtración se toma una alícuota de $20 \mathrm{~mL}$ y se valora con solución de hidróxido de sodio $0,01 \mathrm{~N}$, usando $0,3 \mathrm{~mL}$ de indicador fenolftaleína, observando el cambio de viraje incoloro a rosa.

pH (Método AOAC 981.12): En un vaso de $100 \mathrm{~mL}$ se coloca 1 gramo de muestra de pulpa se añade $50 \mathrm{~mL}$ de agua destilada y se deja en reposo por una hora con agitaciones suaves durante el intervalo de tiempo, finalmente se mide el $\mathrm{pH}$ con un potenciómetro previamente calibrado previamente con tres tipos de buffers 4, 7 y 10 para tener mayor precisión, los valores son reportados con dos decimales. 
Humedad (Método AOAC 930.15): en una balanza analítica se pesa aproximadamente 3 gramos de pulpa y se coloca en una estufa a $100 \pm 5^{\circ} \mathrm{C}$. por 24 horas, al término del tiempo se tapa el recipiente y se coloca en un desecador por un tiempo de 5 minutos, luego se registra el peso final y el porcentaje de humedad se determina por diferencia de pesos.

Cenizas (Método AOAC 942.05): en un crisol de porcelana de $15 \mathrm{~mL}$ de peso conocido, se pesa 2 gramos de pulpa de fruto, luego se coloca en una mufla a $600{ }^{\circ} \mathrm{C}$ por un tiempo de 2 horas, al término del tiempo se retira y coloca en un desecador hasta su enfriamiento registrando su peso final, y por diferencia se calcula el contenido de cenizas.

Proteínas (Método AOAC 984.13) se utilizó el método micro-kjeldhal, para ello se pesa aproximadamente 0,5 gramos de muestra en un tubo del mismo nombre de $50 \mathrm{~mL}$, añadiendo luego 2,5 mL de ácido sulfúrico concentrado, esto se lleva a una plancha de arena hasta solución incolora, se deja enfriar y se destila con hidróxido de sodio al $40 \%$ en un volumen de aproximadamente de $15 \mathrm{~mL}$, el destilado se recibe en un matraz de $100 \mathrm{~mL}$ conteniendo $5 \mathrm{~mL}$ de indicador para proteínas (ácido bórico, rojo de metilo y verde de bromo cresol) hasta un cambio de color rojo a verde, lo cual indica que finalizó el proceso de destilación. Por último, se valora con una solución de ácido clorhídrico de concentración $1 \mathrm{~N}$, hasta un cambio de color rojo. Se calcula el contenido de nitrógeno y se multiplica por el factor 6,25 para expresar el porcentaje de proteína.

Lípidos (Método AOAC 920.039): en un sobre de papel filtro se coloca 3 gramos de pulpa, este se coloca en tubo Soxhlet. Luego en un balón de $250 \mathrm{~mL}$ se añade $160 \mathrm{~mL}$ de solvente hexano, se ensambla el equipo junto con el refrigerante sobre una cocinilla eléctrica, se extrae los lípidos por un tiempo de 2 a 4 horas, al cabo de ese tiempo se retira el sobre y se recupera solvente hasta que solo quede los lípidos extraídos en el balón, luego se lleva a una estufa a $60^{\circ} \mathrm{C}$ para la evaporación completa del solvente, se pesa el balón que contiene la grasa y se determina el contenido por diferencia de peso.

Sólidos solubles (Método AOAC 932.12) modificado, en un vaso de precipitado se coloca 1 gramo de pulpa agregando $50 \mathrm{~mL}$ de agua destilada precalentada a $80^{\circ} \mathrm{C}$, se agita por 2 minutos en un vortex y se deja reposar por un tiempo de 20 minutos, luego se centrifuga a $3000 \mathrm{rpm}$, del cual se toma una alícuota para la medición de los grados ${ }^{\circ} \mathrm{Brix}^{4}$.

\section{Prueba estadística}

Con los resultados obtenidos del análisis físico-químico y proximal encontrados en cada tratamiento se aplicó un análisis estadístico descriptivo teniendo como parámetros principales la media aritmética, desviación estándar y coeficiente de variación $(\mathrm{CV})$, donde un $\mathrm{CV}>50$ $\%$ indica una alta variabilidad y un $\mathrm{CV}<20 \%$ una baja variabilidad según lo expresado por Hidalgo ${ }^{5}$, con un nivel de significancia de 0,05 . 
Tabla 1. Caracteres fisicoquímicos y proximales del fruto de sofaique.

\begin{tabular}{|c|c|c|c|}
\hline \multicolumn{4}{|c|}{ Descripción fisicoquímica y proximal } \\
\hline Análisis & Carácter & Abreviatura & Unidad \\
\hline \multirow{8}{*}{ Físicos } & Peso del fruto & PFRU & \multirow{4}{*}{ g } \\
\hline & Peso de la cáscara & PCAS & \\
\hline & Peso de la pulpa & PPUL & \\
\hline & Peso del carozo & PECAR & \\
\hline & Eje polar & EJEPOL & \multirow{3}{*}{$\mathrm{cm}$} \\
\hline & Eje ecuatorial & EJEECU & \\
\hline & Calibre & CAL & \\
\hline & Ej.pol/ej.ecu & REJPOL/EJECU & \\
\hline \multirow{4}{*}{ Químicos } & Acidez titulable & ACID & Ácido $/ 100 \mathrm{~g}$ \\
\hline & Sólidos solubles & S.S & ${ }^{\circ}$ Brix \\
\hline & $\mathrm{pH}$ & $\mathrm{PH}$ & $\mathrm{pH}$ \\
\hline & Índice de madurez & IMAD & ${ }^{\circ}$ Brix/acidez \\
\hline \multirow{5}{*}{ Proximal } & Humedad & $\% \mathrm{HUM}$ & \multirow{5}{*}{$\%$} \\
\hline & Cenizas & $\% \mathrm{CEN}$ & \\
\hline & Proteínas & $\%$ PRO & \\
\hline & Lípidos & $\%$ LIP & \\
\hline & Carbohidratos & $\% \mathrm{CBH}$ & \\
\hline
\end{tabular}

\section{RESULTADOS Y DISCUSIÓN}

En la tabla 2 se muestra los resultados obtenidos de las pruebas físicas realizadas en el fruto entero de sofaique, las 3 dimensiones promedio fueron: 21,69 mm;22,32 $\mathrm{mm}$ y 19,60 mm para el eje polar, eje ecuatorial y calibre, respectivamente. Con las medias de cada eje se puede presenciar la proximidad en longitud de los mismos, siendo la desviación estándar de sus medias (eje polar, eje ecuatorial y calibre) de 1,42 $\mathrm{mm}$ respecto a la media $21,20 \mathrm{~mm}$.

Por otro lado, la relación del eje polar con el eje ecuatorial tiene un valor de 0,99 lo cual indica que es un fruto con apariencia circular en una de sus caras cuando este valor es próximo a 1. Los resultados obtenidos por Orrabalis ${ }^{6}$ en la región oeste de la provincia Formosa-Argentina (O-Fsa), fue de $28,6 \mathrm{~mm}$ eje polar, $22,9 \mathrm{~mm}$ eje ecuatorial y $20,8 \mathrm{~mm}$ de calibre, donde la relación eje polar/eje ecuatorial resulta 1,25 cuyo valor aduce a un aspecto de forma ovoide, esta diferencia de formas durante el crecimiento hasta su desarrollo final es importante para los procesos de separación de la pulpa y fabricación de máquinas adecuadas acorde a las dimensiones del fruto. 
Tabla 2. Análisis físico del fruto entero de sofaique.

\begin{tabular}{lcccccc}
\hline \multicolumn{1}{c}{ Variable } & Media & DS & R & Mínimo & Máximo & C.V \\
\hline Eje polar & 21,69 & 2,63 & 10,79 & 16,95 & 27,74 & $12,14 \%$ \\
Eje ecuatorial & 22,03 & 2,45 & 9,66 & 16,65 & 26,31 & $11,12 \%$ \\
Calibre & 19,90 & 2,46 & 12,40 & 16,00 & 28,40 & $12,35 \%$ \\
ej.pol/ej.ecu & 0,99 & 0,07 & 0,30 & 0,86 & 1,16 & $6,66 \%$ \\
P. fruto & 3,88 & 1,00 & 3,77 & 1,87 & 5,65 & $25,84 \%$ \\
P. cascarilla & 1,10 & 0,29 & 1,17 & 0,53 & 1,70 & $26,16 \%$ \\
P. pulpa & 1,52 & 0,38 & 1,47 & 0,73 & 2,20 & $25,32 \%$ \\
P. carozo & 1,26 & 0,34 & 1,23 & 0,61 & 1,85 & $27,27 \%$ \\
\hline DS: desviación estándar.C.V: Coeficiente de variación. R: rango & & &
\end{tabular}

El peso promedio del fruto entero fue de 3,88 $\mathrm{g}$ de la pulpa 1,52 $\mathrm{g}$; este valor de la pulpa fue el mayor valor de los tres componentes con una representación del 39,18 \% sobre el peso total del fruto, la cascarilla representa el $28,38 \%$ y el carozo un $32,44 \%$, el CV calculado para los pesos de cada componente del fruto y del mismo fruto fue mayor a $20 \%$ lo cual indica que presenta una moderada variación, en cuanto a la relación cáscara/pulpa fue de 72,43\% en el peso total del fruto. En la región O-Fsa, Orrabalis ${ }^{6}$ determinó un valor promedio para el

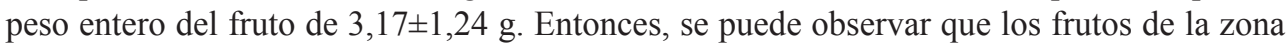
O-Fsa son mayores en dimensión, pero menores en peso, por lo tanto, existe una variación de densidades, donde la región de Ica presenta un valor de $0,393 \mathrm{~g} / \mathrm{cc}$ y la región O-Fsa $0,313 \mathrm{~g} / \mathrm{cc}$, además el porcentaje de presencia del carozo en el fruto en la región de Ica (32 $\%)$ es menor a la región O-Fsa $(40 \%)$. Serrada ${ }^{7}$ indica que los parámetros dimensionales son importantes para determinar y establecer procesos adecuados de: limpieza, secado, calidad, almacenamiento, entre otros. Asimismo, el tamaño, peso y aspecto externo del fruto se verá influenciado por diferentes variables como: naturaleza del suelo, factores climáticos, disponibilidad de nutrientes, variedad y asociación biológica ${ }^{8}$.

En la tabla 3 se presenta los resultados obtenidos del análisis químico del mesocarpio con tres repeticiones por variable, se puede observar que, el porcentaje de acidez resulta ser muy bajo con un valor promedio de 0,09 y una variabilidad de $6,53 \%$; este sería un indicador que los frutos de sofaique tienden a presentar un sabor dulce, además, el tiempo de conservación de los frutos será más prolongado conforme los valores de acidez sean más bajos ${ }^{9}$, asimismo cuando la maduración del fruto aumenta el contenido de ácidos disminuye ${ }^{10}$. 
Tabla 3. Análisis químico de la fracción mesocárpica del fruto de sofaique.

\begin{tabular}{lcccccc} 
Variable & Media & DS & R & Mínimo & Máximo & C.V \\
\hline Acidez & 0,09 & 0,01 & 0,01 & 0,08 & 0,09 & $6,53 \%$ \\
\hline S.S & 4,06 & 0,30 & 0,57 & 3,83 & 4,40 & $7,46 \%$ \\
\hline pH & 5,20 & 0,07 & 0,13 & 5,14 & 5,27 & $1,28 \%$ \\
\hline IMAD & 76,37 & 1,32 & 2,63 & 75,13 & 77,76 & $1,73 \%$
\end{tabular}

Dentro del análisis químico se determinó una concentración para sólidos solubles entre 3,83$4,40^{\circ}$ Brix con una media de $4,06^{\circ}$ Brix, valores que contrastan con lo hallado por Orrabalis ${ }^{4}$, donde determina un rango de 5,9-9,5 ${ }^{\circ}$ Brix para harinas integrales del mismo fruto en diferentes regiones de Argentina. El valor $\mathrm{pH}$ del mesocarpio (pulpa) resultó de 5,2 con una DS de 0,07 y un CV bajo de 1,28 \% resultando ser un valor muy estable. El IMAD promedio del fruto sofaique fue de 76,37 S.S/acidez con un CV de 1,73\%. Al respecto, Galleta et al. ${ }^{11}$ determinaron que la relación entre el nivel de sólidos solubles y acidez es un indicador de la calidad del fruto, considerando que una relación baja de S.S/acidez se asocia a una buena calidad, mientras que valores altos genera indicios de una mayor incidencia de organismos patógenos que pueden causar la pudrición durante el almacenamiento; Hernández ${ }^{12}$ menciona que, en frutos climatéricos el aumento del IMAD se deba probablemente cuando estos alcancen la tasa máxima de respiración, donde ocurre un desdoblamiento de las reservas (ácidos orgánicos), como respuesta al incremento de su metabolismo, es por ello que la acidez decrece en el tiempo para convertirse en azúcar y el índice de madurez aumenta.

Con lo desarrollado se puede inferir que la colecta realizada del fruto de sofaique haya sido tiempo después a la madurez cosechable. Sim embargo Pino ${ }^{13}$ indica que es difícil establecer un solo valor para la relación ss/acidez. dado que existen múltiples combinaciones entre estas variables.

En la tabla 4 podemos apreciar los resultados del análisis proximal del mesocarpio del fruto, con tres repeticiones por variable, donde la humedad promedio resultante fue de $20,2 \%$ con un $\mathrm{CV}$ de $0,62 \%$ lo que indica un valor muy estable; en el estudio realizado por Orrabalis ${ }^{6}$ menciona que el contenido de humedad puede deberse al grado de maduración del fruto y al tipo de clima donde se establece la especie, mencionando lo anterior, se contrasta la precipitación anual de la localidad de Paracas $(0 \mathrm{~mm})$ con la precipitación anual de la región oeste de Formosa $(600 \mathrm{~mm}$ ) donde el contenido de humedad de los frutos es de 18,5 \%; esta excepcional diferencia sobre el contenido de humedad en ambas regiones puede deberse con la proximidad del bosquecillo de sofaique (Paracas) a la cuenca del río Ica, permitiéndole abastecerse del acuífero subterráneo. 
Tabla 4. Análisis proximal de la fracción mesocárpica del fruto de sofaique.

\begin{tabular}{lcccccc}
\hline \multicolumn{1}{c}{ Variable } & Media & DS & R & Mínimo & Máximo & C.V \\
\hline Humedad & 20,20 & 0,13 & 0,25 & 20,09 & 20,34 & $0,62 \%$ \\
Cenizas & 2,59 & 0,06 & 0,11 & 2,55 & 2,66 & $2,22 \%$ \\
Proteínas & 9,17 & 0,54 & 1,08 & 8,65 & 9,73 & $5,88 \%$ \\
Lípidos & 1,89 & 0,71 & 1,24 & 1,07 & 2,31 & $37,53 \%$ \\
Carbohidratos & 66,15 & 0,85 & 1,68 & 65,22 & 66,90 & $1,29 \%$ \\
\hline
\end{tabular}

El promedio de tres ensayos determinó un porcentaje para cenizas de 2,59 \% en el mesocarpio del fruto de sofaique con una desviación estándar de 0,06 ; lo cual hace presencia de un contenido menor de minerales en comparación a lo hallado por Nogués ${ }^{14}$, donde cuantifica un valor de 3,1\% $\%, 09$ para el pericarpio (epicarpio, mesocarpio y endocarpio), asimismo, encontró un valor de $2,9 \% \pm 0,02$ de minerales presente en la semilla de frutos de $G$. decorticans provenientes de zonas áridas y semiáridas de Argentina. Asimismo, Orrabalis ${ }^{4}$ encuentra un rango del contenido de cenizas $(2,5-2,8) \%$ presentes en la harina integral de frutos provenientes de la provincia de Formosa (Argentina) del año 2010, también resalta valores importantes en la integral de sofaique el potasio $(13511,70 \mathrm{mg} / \mathrm{kg})$, el calcio $(657,64$ $\mathrm{mg} / \mathrm{kg}$ ), el magnesio 515,06 mg/kg) siendo una fuente interesante de minerales. Charpentier ${ }^{15}$ determina un contenido de cenizas de $4,3 \% \pm 0,8$ en la provincia Santiago del Estero (Argentina) en el año 1998, por lo tanto, se puede inferir, por medio de los diferentes estudios realizados por los autores ya mencionados, que la variabilidad del contenido de cenizas o sales está sujeto a la disposición de minerales presentes en el suelo y cómo estos disminuyen a lo largo del tiempo, repercutiendo en la composición del fruto.

Uno de los componentes más valorados dentro de la dieta humana es la proteína, lo cual, para la especie $G$. decorticans, su contenido proteico fue de $9,17 \%$ con un coeficiente de variación de 5,9\%,este valor encontrado se asemeja a los valores hallados por Orrabalis ${ }^{6}$ para la harina integral de frutos de sofaique, oscilando valores de 7 a $10 \%$; asimismo, Charpentier ${ }^{15}$ señala un valor de 9,0\% \pm 3 en las zonas áridas y semiáridas de Argentina, por lo cual el contenido proteico se encuentra dentro de los valores normales hallados por los dos autores; además, se puede inferir que estos valores son relativamente mayores a los encontrados en el presente estudio, debido al análisis integral del fruto (pericarpio y semilla) que realizaron los autores en mención. Nogués ${ }^{14}$ separó totalmente el endocarpio de las semillas, encontrando en este último un contenido proteico de $21,6 \% \pm 0,04$; pudiéndose deducir que el componente semilla eleva el contenido porcentual de proteínas en los análisis de frutos enteros. Los porcentajes hallados tienen punto de comparación con algunos alimentos mostrados por el INS como las hojuelas de quinua $(8,5 \%)$, sorgo $(8 \%)$, harina de trigo $(7,9 \%)$, macambo $(6,7 \%)$, etc.; pudiendo estimar que la pulpa del fruto de sofaique presenta un buen contenido proteico.

El contenido de lípidos presentó un bajo valor porcentual de 1,89 \% con un CV de 37,53 \% mucho más alto en comparación con las otras variables y una desviación estándar de 0,71 ; en estudios anteriores realizados en Argentina se determinó un rango para el fruto entero 
de $1,5-2,8 \%$ y $1,42 \%$ de la pulpa, por tanto, el valor calculado se encuentra dentro del rango hallado por Orrabalis ${ }^{9}$ en el consumo humano, el bajo valor de lípidos sería beneficioso para las personas que sufren de sobrepeso, obesidad y diabetes. Para los humanos la parte comestible está constituida por el mesocarpio, que secado al aire contiene alto contenido de carbohidratos (azúcares y fibras). Los altos contenidos de carbohidratos y de azúcares totales de los componentes de la pulpa del fruto de sofaique ofrecerían posibilidades de uso para la preparación de productos alimenticios, del mismo modo los altos valores de azúcares y bajo niveles de grasa, permitirían disponer de una materia prima deseable para elaborar jarabes y mermeladas, entre otros productos.

En cuanto al contenido de carbohidratos, este fue el más alto respecto a las otras variables determinadas con un valor de $66,15 \%$ y un CV mínimo de $1,29 \%$, sin embargo, este valor resulta ser mucho más bajo del determinado por Orrabalis ${ }^{5}$, registrando para el fruto entero un rango de $81,9 \%$ - 87,5\% de carbohidratos totales (azúcares simples, celulosa, fibras y también lignina aportada por el carozo).

\section{CONCLUSIONES}

Por su contenido de humedad junto con su baja acidez, confieren una mayor estabilidad al deterioro del fruto sofaique, evitando el desarrollo de microorganismos. Un fruto de color pardo rojizo, indicaría que este se encuentra en estado maduro. Asimismo, un punto muy importante es el porcentaje elevado de proteínas y carbohidratos que presenta, algo poco común en frutos, lo cual se presenta como una alternativa para la elaboración de productos alimenticios tanto para humanos como para animales.

Para finalizar, las variaciones de los elementos nutricionales disponibles en el suelo influyen considerablemente y de forma directa en el desarrollo del fruto de sofaique y en su composición química.

\section{AGRADECIMIENTOS}

La presente investigación se realizó en las instalaciones del Laboratorio de Pulpa y Papel del área de Transformación Química de la Madera de la Facultad de Ciencias Forestales de la UNALM. Al Técnico de Laboratorio Luis E. Cabrera Vaquerizo y a las estudiantes de la carrera de Ciencias Forestales, Alessandra Crisanto Ventura y Raquel Rivera Canales quienes contribuyeron en el desarrollo de la presente investigación. 


\section{REFERENCIAS BIBLIOGRÁFICAS}

1. Robertson CE. Book review: McCance and Widdowson's the composition of foods sixth summary edition. Nutr Bol. 2003; 28(1): 81-83.

2. INRENA. Mapa Ecológico del Perú. Lima, Perú. [Internet]. 1995 [Citado 10 Jul 2020]. Disponible en: http://www.keneamazon.net/Documents/Publications/Virtual-Library/ Maps/INRENA-mapa-ecologico.pdf

3. AOAC. Association of Offical Analytical Chemistry. $18^{\text {th }}$ Ed. Gaithersburg, MD (Estados Unidos): AOAC International; 2005.

4. Orrabalis CJ, Gorostegui H, Calandri E, Guzmán C. Estudio sobre parámetros funcionales y contenido de glucosa en harinas obtenidas de fruto maduro de "Chañar" de la zona semiárida y árida de la provincia de Formosa. Multequina [Internet]. 2013 [citado 12 Jul 2020]. 22. 2013. Disponible en: https://www.researchgate.net/ publication/279317953_Chemical_characterization_and_sensory_evaluation_of_new_ sweets_made_with_Geoffroea_decorticans_fruits_Fabaceae

5. Franco TL, Hidalgo R. Variabilidad Genética y Caracterización de Especies Vegetales: Análisis estadísticos de datos de Caracterización Morfológica de recursos Fitogenéticos. Bol Tec IPGRI, No 8. [Internet]. Cali, Colombia: Instituto Internacional de Recursos Fitogenéticos; 2003. [Citado 21 Jun 2020]. Disponible en https://www. bioversityinternational.org/fileadmin/_migrated/uploads/tx_news/An\%C3\%A1lisis_ estad\%C3\%ADstico_de_datos_de_caracterizaci\%C3\%B3n_morfol\%C3\%B3gica_de_ recursos_fitogen $\% \mathrm{C} 3 \%$ A9ticos_894.pdf

6. Orrabalis CJ. Aprovechamiento Integral de los Frutos de Geoffroea decorticans (chañar), de la Región Fitogeográfica de la Provincia de Formosa. [Tesis doctoral]. Córdova, Argentina: Universidad Nacional de Córdoba; 2014.

7. Serrada R. Apuntes de Repoblaciones Forestales: Generalidades sobre semillas forestales. FUCOVASA. [Internet] Madrid; 2000. [Citado 28 Jun 2020]. Disponible en: https://www.uv.mx/personal/tcarmona/files/2019/02/Serrada-2000.pdf

8. Romojaro F, Martínez M, Pretel M. Factores precosecha determinantes de la calidad y conservación en poscosecha de productos agrarios. [Internet]. 2007. [Citado 28 Jun 2020]. Disponible en: https://www.researchgate.net/publication/237402419_Factores precosecha_determinantes_de_la_calidad_y_conservacion_en_poscosecha_de_ productos_agrarios

9. Rodríguez D, Patiño M, Miranda D, Miranda G, Galvis J. Efecto de dos índices de madurez y dos temperaturas de almacenamiento sobre el comportamiento en almacenamiento sobre el comportamiento en postcosecha de la pitahaya amarilla (Selenicereus megalanthus haw,). Rev Facu Nac Agron. 2005; 58(2): 2837-2857.

10. Salgado T, Martinez R. Relación entre la intensidad respiratoria y las propiedades fisicoquímicas del banano (Musa sapientum 1) var, criollo, tomate de árbol (Solamun betaceum) var, morada y mango (Mangifera). [Tesis de Bachiller]. Bogotá: Facultad de Ingeniería de Alimentos, Universidad de La Salle; 2006.

11. Galletta G; Ballinger W; Monroe R, Kushman L. Relationships between fruit acidity and soluble solids levels of highbush blueberry clones and fruit keeping quality. Amer Soc Hort Sci J. 1971; 96 (6): 758-762. 
12. Hernández MS. Conservación del fruto del arazá (Eugenia stipitata) durante la poscosecha mediante la aplicación de diferentes técnicas. [Tesis doctoral]. Bogotá: Facultad de Agronomía, Universidad Nacional de Colombia; 2001.

13. Pino C. Descripción del desarrollo vegetativo y de las características físicas y químicas de los frutos de cuatro clones de arándano alto (Vaccinium corymbosum L.). [Tesis de Bachiller]. Valdivia, Chile: Facultad de Agronomía. Universidad Austral de Chile; 2007.

14. Nogués E, Castro O, Correa R, Puricelli M, Gómez H, Beale I, et al. Revalorización del chañar (Geoffroea decorticans) en ambientes áridos y semiáridos de Argentina. Rev Divul Téen Agrí Agroin. [Internet]. Jun 2013. [Citado 15 Jul 2020]. 45. Disponible en: http://agrarias.unca.edu.ar/wp-content/uploads/2018/Revista\%20 de\%20Divulgaci\%C3\%B3n\%20T\%C3\%A9cnica\%20Agr\%C3\%ADcola\%20y\%20 Agroindustrial/Revista-45-Revalorizaci\%C3\%B3n-del-Cha\%C3\%B1 ar-en-losambientes-\%C3\%A1ridos.pdf

15. Charpentier M. Valores Nutricionales de las Plantas Alimenticias Silvestres del Norte Argentino. Santa Fe, Argentina: INCUPO; 1998. 